\title{
Impact of Geoid Improvement on Ocean Mass and Heat Transport Estimates
}

\author{
Pascal Le Grand
}

IFREMER, BP 70, F-29280 Plouzane, France

plegrand@ifremer.fr

\begin{abstract}
:
One long-standing difficulty in estimating the large-scale ocean circulation is the inability to observe absolute current velocities. Both conventional hydrographic measurements and altimetric measurements provide observations of currents relative to an unknown velocity at a reference depth in the case of hydrographic data, and relative to mean currents calculated over some averaging period in the case of altimetric data. Space gravity missions together with altimetric observations have the potential to overcome this difficulty by providing absolute estimates of the velocity of surface oceanic currents. The absolute surface velocity estimates will in turn provide the reference level velocities that are necessary to compute absolute velocities at any depth level from hydrographic data.

Several studies have been carried out to quantify the improvements expected from ongoing and future space gravity missions. The results of these studies in terms of volume flux estimates (transport of water masses) and heat flux estimates (transport of heat by the ocean) are reviewed in this paper. The studies are based on ocean inverse modeling techniques that derive impact estimates solely from the geoid error budgets of forthcoming space gravity missions. Despite some differences in the assumptions made, the inverse modeling calculations all point to significant improvements in estimates of oceanic fluxes. These improvements, measured in terms of reductions of uncertainties, are expected to be as large as a factor of 2.

New developments in autonomous ocean observing systems will complement the developments expected from space gravity missions. The synergies of in situ and satellite observing systems are considered in the conclusion of this paper.
\end{abstract}




\title{
IMPACT OF GEOID IMPROVEMENT ON OCEAN MASS AND HEAT TRANSPORT ESTIMATES
}

\author{
P. LE GRAND (plegrandeifremer.fr) \\ IFREMER, BP 70, F-29280 Plouzane, France
}

Received: 17 May 2002; Accepted in final form: 15 November 2002

\begin{abstract}
One long-standing difficulty in estimating the large-scale ocean circulation is the inability to observe absolute current velocities. Both conventional hydrographic measurements and altimetric measurements provide observations of currents relative to an unknown velocity at a reference depth in the case of hydrographic data, and relative to mean currents calculated over some averaging period in the case of altimetric data. Space gravity missions together with altimetric observations have the potential to overcome this difficulty by providing absolute estimates of the velocity of surface oceanic currents. The absolute surface velocity estimates will in turn provide the reference level velocities that are necessary to compute absolute velocities at any depth level from hydrographic data.

Several studies have been carried out to quantify the improvements expected from ongoing and future space gravity missions. The results of these studies in terms of volume flux estimates (transport of water masses) and heat flux estimates (transport of heat by the ocean) are reviewed in this paper. The studies are based on ocean inverse modeling techniques that derive impact estimates solely from the geoid error budgets of forthcoming space gravity missions. Despite some differences in the assumptions made, the inverse modeling calculations all point to significant improvements in estimates of oceanic fluxes. These improvements, measured in terms of reductions of uncertainties, are expected to be as large as a factor of 2 .

New developments in autonomous ocean observing systems will complement the developments expected from space gravity missions. The synergies of in situ and satellite observing systems are considered in the conclusion of this paper.
\end{abstract}

\section{Introduction}

As it is more and more evident that human activities have a significant impact on the climate system (IPCC, 2001), it becomes highly desirable to improve our capability to observe, quantify, and predict climate change. The ocean is an important component of the climate system through its role in redistributing heat from equatorial regions to polar regions (Ganachaud and Wunsch, 2000) and through its role in regulating the carbon cycle (Sigman and Boyle, 2000). In turn, climate change can potentially affect the ocean circulation and chemistry through various feedback mechanisms (Sigman and Boyle, 2000). Observations of the ocean have until recently been scarce, not the least because of the difficulty of collecting data in this high-pressure, corrosive, opaque, and generally hostile environment. The past decade, however, has seen the advent of precise satellite observing systems like space-borne altimeters, which for the first time have allowed physical oceanogra- 
phers to observe the ocean circulation in a global and quasi-synoptic way. Two major difficulties remain, however, in using altimetric data to quantify the ocean circulation and the processes that are associated to it. The first difficulty is that altimetric observations only sample the surface of the ocean. In situ measurements are therefore required to constrain the interior, the circulation being highly variable from the surface to the bottom of the ocean. In the oceanographic terminology, the ocean circulation is said to contain a strong baroclinic, or non-barotropic, component. The interpretation of hydrographic measurements in terms of baroclinic circulation are developed in section 2 of this paper.

The second difficulty in using altimetric data to quantify the ocean circulation is that these data do not constrain the ocean circulation directly. Instead, they constrain the sum of the geoid height and of the ocean dynamic topography, so it is difficult to disentangle the two signals. This problem is reviewed in section 3.

In the near future, dedicated space gravity missions like GOCE and GRACE will allow a precise determination of the geoid height and will thus allow the separation of the ocean circulation signal from the geoid signal in altimetric observations. Resulting improvements in circulation estimates are reviewed in section 4 of this paper, with a particular attention on volume and heat transports that are two quantities of great importance in the climate system. The last section of this paper concludes by investigating the complementarities between observations provided by the forthcoming space gravity missions and new automated in situ measurement techniques that are now becoming available on the global scale. The complementarities of the GOCE and the GRACE gravity missions are also considered in this section.

\section{Hydrographic Measurements and Ocean Circulation}

In situ measurements that constrain the baroclinic ocean circulation are available in the form of hydrographic observations. These observations consist of temperature and salinity measurements collected at a known pressure level using a Conductivity, Temperature, Depth (CTD) instrument lowered from an oceanographic vessel. The data are thus collected as vertical profiles at a station above a more or less fixed point. Using the equation of state of sea water (Fofonoff, 1985), temperature and salinity are then converted into density. Density is in turn related to the baroclinic ocean circulation through the thermal wind relations:

$$
f \frac{\partial u}{\partial z}=+\frac{g}{\rho} \frac{\partial \rho}{\partial y} \quad ; \quad f \frac{\partial v}{\partial z}=-\frac{g}{\rho} \frac{\partial \rho}{\partial x}
$$

where $u$ and $v$ are the horizontal components of the velocity field, $f$ is the coriolis parameter, $\rho$ is the density, $g$ is the gravitional acceleration, $x$ and $y$ the horizontal coordinates, and $z$ is the vertical coordinate. These equations are derived (see Pond and Pickard, 1983) from 1) the geostrophic balance, which assumes 
that the dominant terms in the horizontal momentum equations are the Coriolis force and the pressure force 2) the hydrostatic balance, which assumes that vertical gradients of pressure are proportional to density. These approximations are very good ones in the ocean, and deviations from them are in most areas unmeasurably slight (Wunsch, 1996). The main exceptions, where the approximations completely fail, occur at the equator where the Coriolis parameter vanishes and near the surface of the ocean where a large fraction of the velocity field is directly caused by the transfer of momentum from the atmosphere to the upper ocean. The terminology "thermal wind" comes from meteorology because in the atmosphere density can be replaced by temperature using the law of ideal gases. Note that, because of the simplifications made, the thermal wind equations, as well as the geostrophic and hydrostatic equations, are not self-contained. Indeed, unlike the full-blown NavierStokes equations, which can in principle be solved if initial/boundary conditions are known, the thermal wind relations require observational information in order to determine the ocean circulation. For this reason, the thermal wind equations are said to be diagnostic as opposed to the Navier-Stokes equations, which are said to be prognostic. Integrating (1) with respect to $z$, one obtains:

$$
u(h)=u_{r}+\int_{z_{r}}^{h} \frac{g}{f \rho} \frac{\partial \rho}{\partial y} d z \quad ; \quad v(h)=v_{r}+\int_{z_{r}}^{h} \frac{-g}{f \rho} \frac{\partial \rho}{\partial x} d z
$$

where $u(h)$ and $v(h)$ are the horizontal velocities at any depth level $h$, and $u_{r}$ and $v_{r}$ are the velocities at the arbitrarily chosen depth $z_{r}$. One sees from (2) that horizontal velocities at depth level $h$ are determined from the density of sea water, up to the arbitrary constants $u_{r}$ and $v_{r}$. These constants are usually called reference level velocities, and $z_{r}$ is called the reference level. In the early days of oceanography, it was common to make the assumption that, provided that the reference level was chosen deep enough or in between water masses that flow in opposite directions, the reference level velocities could be neglected. In the 1970s, this traditional assumption was shown to be far from valid, and more rigorous approaches had to be designed. This led to the introduction of inverse methods in oceanography (Wunsch, 1978) which use available information to estimate the reference level velocities instead of neglecting them. This available information is quite eclectic (Wunsch, 1984). It comes, for instance, from the constraint that mass should be conserved within closed volumes in the ocean. If one considers a transAtlantic hydrographic section at $24^{\circ} \mathrm{N}$, for instance, the reference level velocities must be consistent with the constraint that the net flux of mass across this section is close to zero. Indeed, any large flux would have to be balanced by large sources or sinks of water that are not observed north of the section (in this example, it is assumed, for the sake of simplicity, that the net flux of water across the Bering Strait and the net air-sea flux of water over the North Atlantic are negligible). If the oceanic section is discretized onto a number of gridpoints, the constraint of zero 
net flux can be formalized as a set of linear equations that relates discrete reference level velocities to the observations:

$$
\mathbf{A x}=\mathbf{b}
$$

where $\mathbf{A}$ is a known matrix, $\mathbf{x}$ is a vector containing the unknown reference level velocities at each gridpoint, and $\mathbf{b}$ is a known vector containing zeros or, more generally, quantities known from observations. Most often, the number of equations is smaller than the number of unknowns in (3), so the problem is underdetermined. Moreover, the equations contained in (3) are never fully exact because of discretization errors and, more importantly, because of observational uncertainties. For the mass conservation equation associated with the $24^{\circ} \mathrm{N}$ section, for instance, the net flux of water across the Bering Strait is known to be small but not exactly zero (Coachman and Aagaard, 1988), so one cannot strictly impose that the flux of water across $24^{\circ} \mathrm{N}$ is zero. Formally, one should therefore replace (3) by the following equation:

$$
\mathbf{A x}=\mathbf{b} \pm \mathbf{n}
$$

where $\mathbf{n}$ is a vector containing the unknown errors in the constraints. Thus, (4) is always underdetermined. It can still be solved, however, using some variant of least-squares techniques that, for instance, minimizes the departure from a known prior estimate. Obviously, the least-squares solution will be subject to uncertainties, but these uncertainties can also be computed as part of the least-squares procedure so that one can attribute a degree of confidence to the solution.

Once the reference level velocities have been estimated, the oceanic transports of volume, or of any other property, can be computed using (2). The ocean inverse estimation procedure can also be implemented over a gridded model. In this case, the mass conservation constraints, or any other type of constraints, are written locally for each box defined by the grid of the model. The available constraints are numerous, ranging from dynamical constraints such as mass and heat conservation, to observational constraints other than hydrographic observations (current meter moorings that measure velocities at a fixed point in the ocean, for instance). Satellite altimetry is one of the newest, and potentially one of the most powerful, observational constraints. How these remote sensing observations can be implemented in the eclectic inverse procedure described above is the focus of the following section.

\section{Altimetric Constraints and the Ocean Circulation Estimation Problem}

Altimetric observations constrain the sea surface height, which is the sum of 1) the ocean dynamic topography caused by surface oceanic currents and 2) the geoid height that corresponds to the elevation the sea surface would have if the ocean were at rest. Currently available estimates of the geoid height, which is by far 
the dominant signal, are not precise (errors of several 10's of $\mathrm{cm}$ at the spatial scales of 1 degree that characterize intense oceanic currents such as the Gulf Stream (Lemoine et al., 1998)). It is thus difficult to remove the geoid signal from the sea surface height observations in order to precisely estimate the residual dynamic topography, which is the quantity of interest to physical oceanographers. Note, however, that the time-varying component of the ocean circulation can be determined because temporal variations in the Earth gravity field are negligible compared to temporal variations in the ocean dynamic topography. Altimetric observations are easily implemented in the inverse estimation procedure. Indeed, these observations provide constraints on velocities at the surface of the ocean through the geostrophic relations:

$$
f u(0)=-g \frac{\partial \eta}{\partial y} \quad ; \quad f v(0)=+g \frac{\partial \eta}{\partial x}
$$

where $\eta$ is the ocean dynamic topography and $u(0)$ and $v(0)$ are the geostrophic velocities at the surface of the ocean. Non-geostrophic Ekman velocities caused by the direct effect of wind forcing are also present at the surface of the ocean (Pond and Pickard, 1983). It is assumed in the following that the volume transport associated with these velocities can be independantly estimated so that it does not limit the precision of overall transport estimates. Although this assumption is not valid at present, there is good hope that accurate ocean surface wind fields will become available in the near future through the assimilation of satellite scatterometer observations in atmospheric general circulation models (see the Question and Answer section in Milliff et al. (2001) for a discussion of this point). Note that the improvements in transport estimates presented below would largely remain valid for the geostrophic component of the velocity field even in the absence of improvements in the Ekman component.

A discretized version of equation (5) provides observational constraints on surface velocities which can easily be added to the set of linear equations (4). Because they are mostly due to errors in the geoid height model that are spatially correlated, uncertainties in the altimetric constraint must be represented by a full covariance matrix. At present, this constraint moderately improves the inverse solution, mostly in the Southern Ocean (LeGrand et al., 2002). It has not yet, however, reached its full potential. It will do so when precise geoid models provided by space gravity missions like GOCE and GRACE, and to some extent CHAMP, become available. The expected improvements are reviewed in the following section.

\section{Impact of Geoid Improvement on Ocean Mass and Heat Transport Estimates}

With improved estimates of the geoid, the conversion of altimetric data into absolute dynamic topography will become possible. Using this information, oceanographers will be able to precisely estimate the circulation at the surface of the ocean. 
These improved surface estimates, combined with in situ hydrographic measurements, will in turn provide strong constraints on the ocean circulation at all depths. The resulting improvements can be quantified in terms of reduction of the uncertainties in the least-squares solution of (4) without knowing the actual geoid height provided by the gravity missions.

Following this approach, improvements in oceanic transport estimates have been quantified in three inverse modelling studies using the expected precision of future gravity missions. Two of them (Ganachaud et al., 1997; Schröter et al., 2002) use box inverse models in which oceanic transports are calculated across transoceanic sections, whereas the third one (LeGrand, 2001) uses a finite difference inverse model. Wunsch and Stammer (this volume) also show how improved geoid measurements can be used in an ocean data assimilation framework to improve both estimates of the ocean circulation and the geoid itself. Inverse modelling and data assimilation techniques are conceptually very similar, although a stronger emphasis is put on the dynamical constraints in the second approach. The most notable advantage of considering a more complex dynamical framework is the inclusion of time-dependent processes. For that reason, the data assimilation approach will allow the complete interpretation of the time-dependent observations of the gravity field provided by the GRACE mission. (see the article by Nerem in this volume for a discussion of the time-varying effects resolved by GRACE.) The approach described here has the advantage of being easy to implement, and to provide estimates of the uncertainty in the solution.

The Ganachaud et al. (1997) calculation studies the impact of three different hypothetical low-low satellite-to-satellite tracking missions, the specifications of the third mission including GPS tracking and resembling the specifications of the GRACE mission. In Ganachaud et al.'s (1997) calculation, only the reference velocities are treated as variables and therefore the velocity uncertainties due to the terms of equations (2) proportional to the ocean density field are not explicitly taken into account. In the calculation corresponding to a GRACE-like mission (specifications provided for a spherical harmonic expansion up to degree 70), the density uncertainties are set to zero in order to explore the best possible outcome. For that reason, the results of Ganachaud et al. (1997) shown in Table I can be considered as an optimistic estimate of the impact of GRACE based on the assumption that all errors in the in situ density field could be rendered negligible by the use of a network of simultaneous and precise in situ observations. These optimistic assumptions are not justified if the objective is to estimate transport uncertainties in layers of the ocean because transports calculated over a limited depth range are often dominated by the density term of equation (2) and thus the uncertainties in these transports are controlled by the uncertainties in the density field. The assumptions can be partly justified, however, when the objective is to estimate the uncertainties in transports integrated from the surface to the bottom of the ocean. Indeed, reference level velocities often dominate vertically integrated transports across trans-oceanic sections because, despite being small, they translate 
TABLE I

Results of various impact studies.

\begin{tabular}{llll}
\hline Study & $\begin{array}{l}\text { Ganachaud et al. (1997) } \\
\text { (heat fluxes, Bettadpur } \\
70 \text { case) }\end{array}$ & $\begin{array}{l}\text { LeGrand (2001) (Vol- } \\
\text { ume fluxes in the upper } \\
\mathrm{km} \text { of the ocean) }\end{array}$ & $\begin{array}{l}\text { Schröter et al. (2002) } \\
\text { (heat flux in section } \\
\text { across the Gulf Stream } \\
\left.\text { at } 24^{\circ} \mathrm{N}\right)\end{array}$ \\
\hline $\begin{array}{l}\text { Transport } \\
\begin{array}{l}\text { uncertainty } \\
\text { reduction }\end{array}\end{array}$ & $1 \%$ to $60 \%$ & $\begin{array}{l}7 \% \text { to } 42 \% \text { for GOCE } \\
\text { and } 2 \% \text { to } 20 \% \text { for } \\
\text { GRACE }\end{array}$ & $\begin{array}{l}37 \% \text { for GOCE and } 29 \% \\
\text { for GRACE }\end{array}$ \\
\hline
\end{tabular}

into large transports when integrated over the whole water column. Note that the uncertainties in volume transports across trans-oceanic sections closing an ocean basin are likely to be very small, even without the altimetric/geoid constraint on dynamic topography. Indeed, volume transports across these sections cannot significantly deviate from zero, as in the example of the $24^{\circ} \mathrm{N}$ section, because there is no large net flux of volume across the northern boundary of the basins closed by these sections. It is therefore not surprising that Ganachaud et al. (1997) find that mass* transport uncertainties are usually not reduced much for the sections that close an ocean basin. Accordingly, they find that the largest impact occurs in the open Southern Ocean basin. The same argument cannot be applied to the case of heat transports because these transports are largely caused by the different heat contents of surface waters flowing in one direction and deep waters flowing in the opposite direction (Figure 1). Thus, heat transports can be large across a section, even if the corresponding net volume transport is equal to zero. Ganachaud et al. (1997) find a reduction in heat flux uncertainties that range between negligible values in some areas of the North Atlantic and values of $60 \%$ in the South Pacific/Indian Oceans and in the Circumpolar Current (Table I).

A different impact calculation is presented in LeGrand (2001). In this study, the uncertainties in the density field are explicitly taken into account so that the impact of gravity missions on transport uncertainties can be estimated for various layers of the ocean. The impact of the GOCE and GRACE missions is estimated across various sections of the Atlantic Ocean, including sections across narrow oceanic currents like the Gulf Stream, the Azores Current, and the Benguela Current. The Atlantic Ocean contains most of the processes active in the ocean, including deep water formation, boundary currents, and wind driven gyres so the results of the study are fairly general despite the limited geographical extent of the model domain. Because temperature is not an explicit variable in the inverse model, only the

* Mass transports and volume transports describe the same processes. Mass transports are more relevant because mass is strictly conserved in the ocean but not volume. However, replacing the condition of non-divergence of mass fluxes by a condition of non-divergence of volume fluxes is a good approximation almost everywhere. 


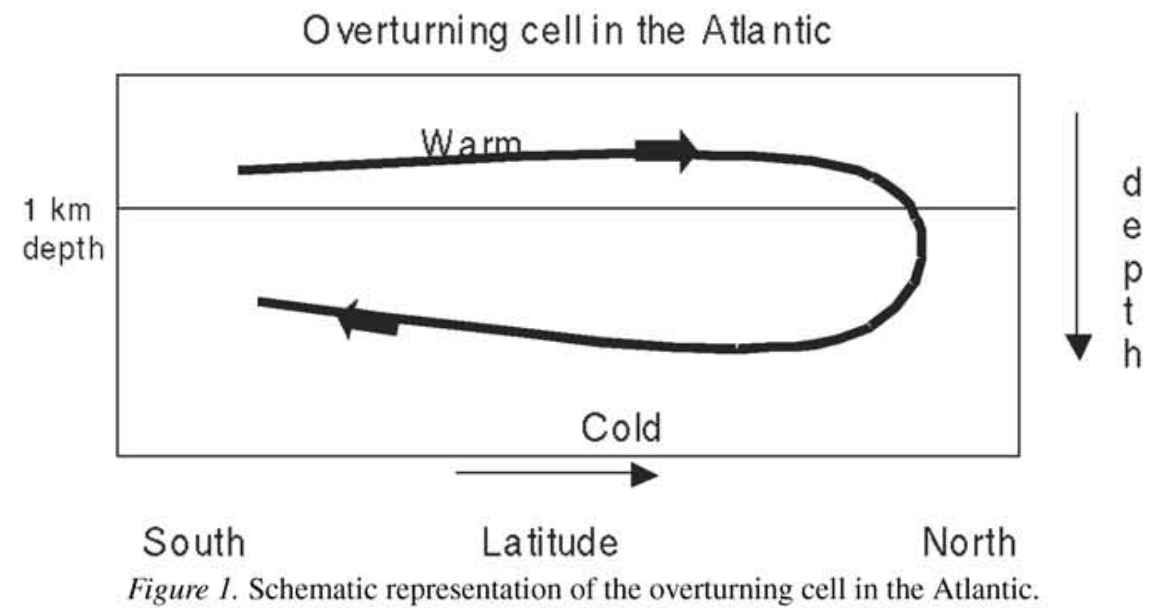

impact of gravity missions on volume fluxes is investigated. However, meridional heat fluxes in the Atlantic are conditioned to a large extent by the overturning cell (Hall and Bryden, 1982) by which the northward flow of warm waters in the upper layers of the ocean is compensated by a return flow of cold waters in the deep layers of the ocean (Figure 1). Thus, by looking at the impact of gravity missions on volume fluxes in the upper kilometer or so, one gets a good idea of how heat fluxes will be improved. The uncertainty reductions obtained by LeGrand (2001) are shown in Figure 2 for the upper kilometer of the ocean, and summarized in Table I. In the GOCE calculation, they range from small improvements across trans-oceanic sections to improvements larger than $40 \%$ in the Brazil Current and in the Circumpolar Current. In absolute terms, the uncertainty reductions reach several Sverdrups ( 1 Sverdrup $\left.=10^{6} \mathrm{~m}^{3} / \mathrm{s}\right)$. In the GRACE calculation, the impact is, on average, slightly less than half the impact of GOCE because GRACE will not resolve the small spatial scales of the ocean circulation. Note that the inverse calculation is a climatological one so the temporal variations that GRACE intends to observe are not investigated. The main conclusion of the study is that, when the uncertainties in the density field are taken into account, the impact of gravity missions on oceanic transport estimates is smaller at depths. This result is not surprising since the combination of altimetry and gravity observations will provide information on the circulation at the surface of the ocean, and the transmission of this information to the deep layers of the ocean will be hampered by the presence of noise in the in situ observations of the density field. The main contribution of gravity missions will thus be an improvement in transport estimates in the upper branch of the meridional overturning cell shown schematically in Figure 1.

The box-inverse model impact study of Ganachaud et al. (1997) has been revisited by Schröter et al. (2002) in view of the characteristics recently specified for GOCE and GRACE. Moreover, Schröter et al. (2002) investigate the potential impact of gravity missions across a trans-oceanic section, the $24^{\circ} \mathrm{N}$ one mentionned 

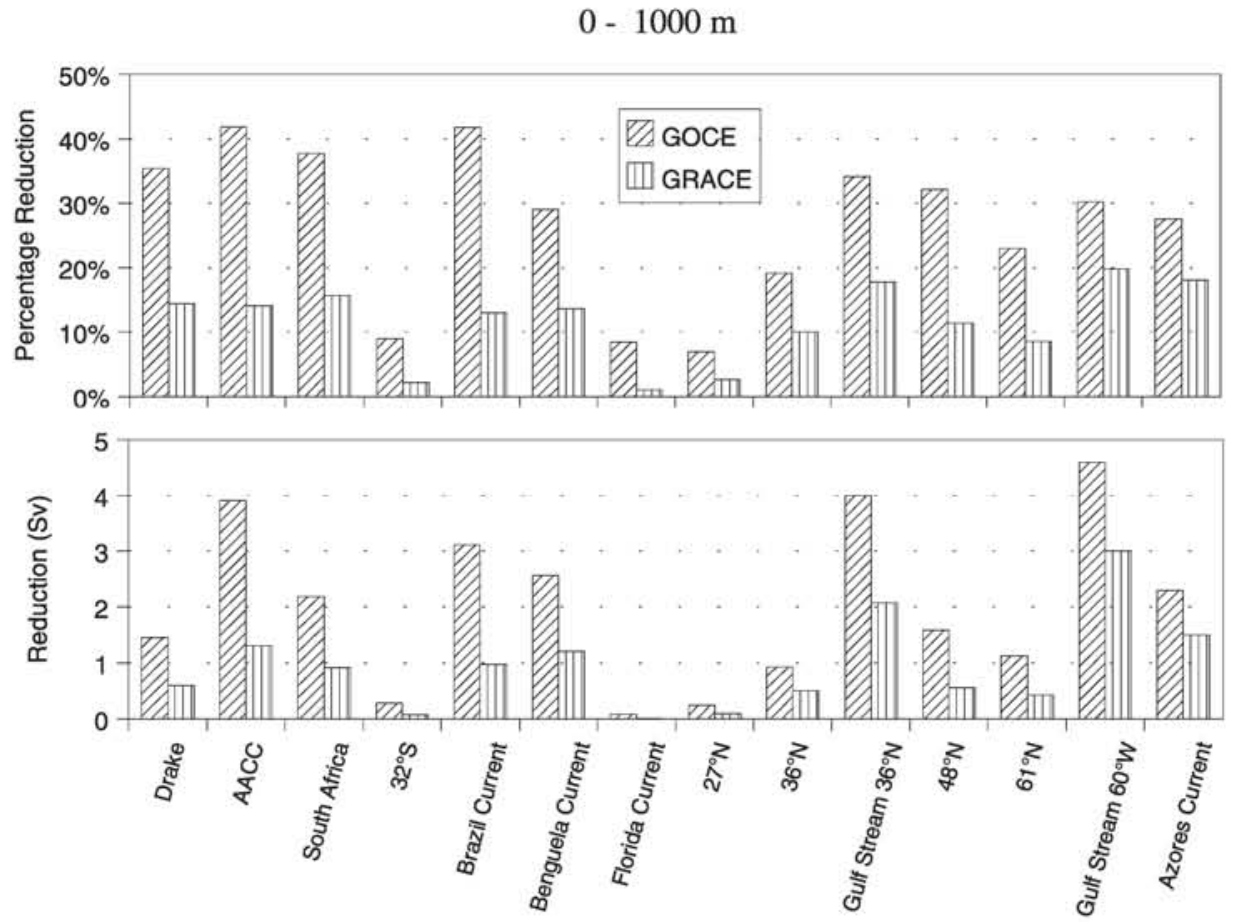

Transports Estimated with EGM96

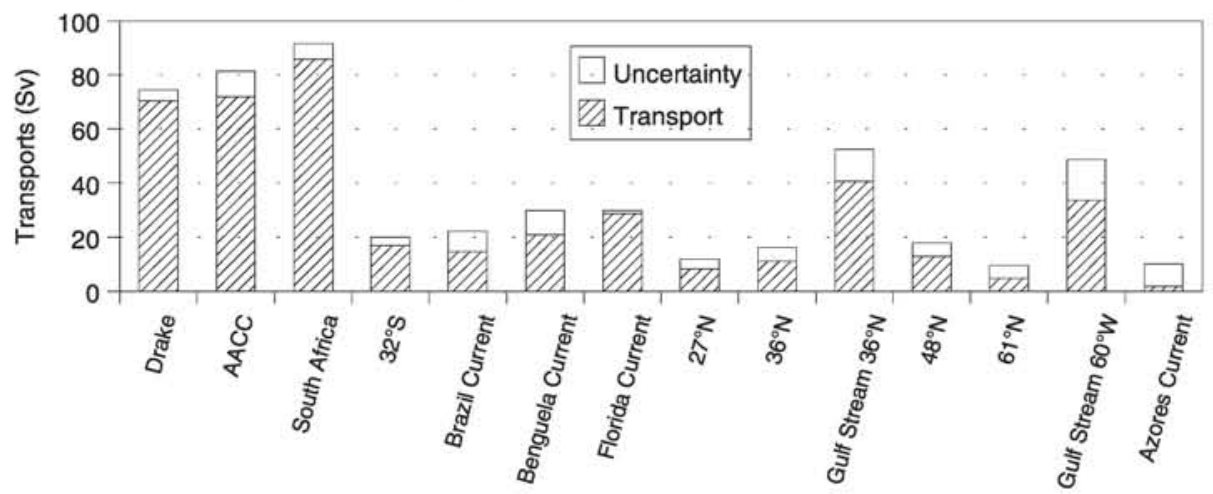

Figure 2. Impact of the GOCE and GRACE gravity missions on volume transports in the upper kilometer of the ocean. (top) Percentage reduction of transport uncertainties relative to EGM96 reference uncertainties. (middle) Corresponding absolute reduction in Sverdrups. (bottom) Transports and associated uncertainties found in the reference EGM96 calculation. 
in the example of section 2, using a fully non-linear inverse model constrained by pseudo in situ observations generated by a general circulation model of the ocean circulation. In this study, uncertainties in the temperature and in the salinity fields are explicitly considered and all the contributions to the uncertainties in heat fluxes are taken into account. The results obtained by Schröter et al. (2002) confirm the results obtained in previous studies with a reduction of heat flux uncertainties of $37 \%$ for GOCE and 29\% for GRACE in the Gulf Stream area (Table I). The pseudo in situ observations used in this study introduce the idea of synoptic high quality observations of the ocean. The following section will show that obtaining this kind of observations in the real ocean will become possible in the near future.

\section{Complementarities of Space Gravity Missions and Other Observing Techniques}

Ideally, space gravity observations and other observations should all be obtained simultaneously for consistency. Because the marine geoid height evolves slowly, it can be considered to be constant relative to the fast pace at which other quantities evolve. Thus, once the geoid has been estimated, it can be used for many years in order to derive the absolute dynamic topography of the ocean from altimetric observations. However, the previous sections showed that altimetric observations of the surface of the ocean must be combined with in situ observations in order to estimate the ocean circulation at all depths. The problem is that in situ observations have so far been difficult and expensive to obtain. Oceanographers have thus relied on climatologies that average hydrographic observations over many years in order to get a reasonably good spatial coverage. This averaging, unfortunately, prevents the estimation of the instant ocean circulation. Until recently, all that could be obtained at basin scale was an estimate of the "mean ocean circulation" over the many years spanned by the climatology.

This situation is about to change with the advent of ocean in situ observing networks. These networks, inspired by the network of sounding balloons used in meteorology, consist in a large number of profiling floats that drift in the ocean at a parking depth that can be as deep as $2000 \mathrm{~m}$. At the end of pre-programmed periods, which range from a few days to a few weeks, the floats go back up to the surface of the ocean. While doing so, they sample vertical profiles of temperature and salinity. Once surfaced, the floats transmit the data to a ground station through a satellite connection. The float network can thus sample the interior of the ocean at regular intervals, just like altimetric missions sample the surface of the ocean every 10 to 30 days. When a complete network of floats is deployed (Figure 3 ), oceanographers will be able to map the hydrographic properties of the ocean in a quasi-synoptic way. Such a network is currently being deployed in the framework of the international ARGO program (http://www-argo.ucsd.edu; Le Traon, this volume) and should be available before the launch of the GOCE mission. 
The hydrographic fields, just like traditional shipborne data, will provide velocity estimates relative to a reference level velocity.* Space gravity missions, by allowing the interpretation of altimetric observations in terms of absolute surface velocities will then provide the complementary information on reference level velocities. The uncertainties in the density field will be reduced because the noise introduced in the climatological estimates by the ocean natural variability will no longer be present in the quasi-instantaneous float observations. One may thus hope that the transmission of the very precise information provided by satellite observations to the deep layers of the ocean will no longer be hampered by the uncertainties in the density field. The challenge, however, will be to deploy an observing network that has a resolution sufficient to resolve the spatial scales of the large-scale ocean circulation. If the resolution of the network is not high enough, the data can still be assimilated in general circulation models in order to provide analyzed density fields, similar to meteorological re-analyses. The inverse approach will then serve to estimate not only the velocity field, which can be done directly by the data assimilation procedure, but also its uncertainty, which will remain out of reach of the data assimilation approach in the near future.

One final point is that the various gravity missions already undergoing or planned for the near future will also be complementary. GOCE, combined with altimetry, will provide precise estimates of the absolute ocean circulation at the surface. GRACE will provide estimates of temporal variations of bottom pressure signals at the bottom of the ocean. The difference in bottom pressure sampled by GRACE on both sides of any trans-oceanic section will provide a constraint on the temporal variations of the integrated geostrophic transport across that section. Indeed, the formulation of the geostrophic balance at depth is:

$$
f u(h)=-\frac{1}{\rho} \frac{\partial p}{\partial y} \quad ; \quad f v(h)=+\frac{1}{\rho} \frac{\partial p}{\partial x}
$$

where $p$ denotes the pressure at depth $h$. Integrating the equation for the meridional velocity for instance, one obtains:

$$
f \int_{x_{e}}^{x_{w}} v(h) d x=f T=\frac{1}{\rho}\left(p_{e}-p_{w}\right)
$$

where $T$ is the zonally integrated transport per unit depth and $p_{e}$ and $p_{w}$ are the bottom pressures measured by GRACE at the extremities of the section (longitudes $x_{e}$ and $x_{w}$ ). By providing a series of bottom pressure measurements along the bathymetric line, GRACE will constrain the deep oceanic transports that are the least-constrained by GOCE.

* Float displacements during the drifting period can in principle be used to estimate absolute current velocities but the interpretation of these displacements is difficult because the amount of drift occurring while the float is at the surface of the ocean can be as large as the drift occurring while the float is at its parking depth. 

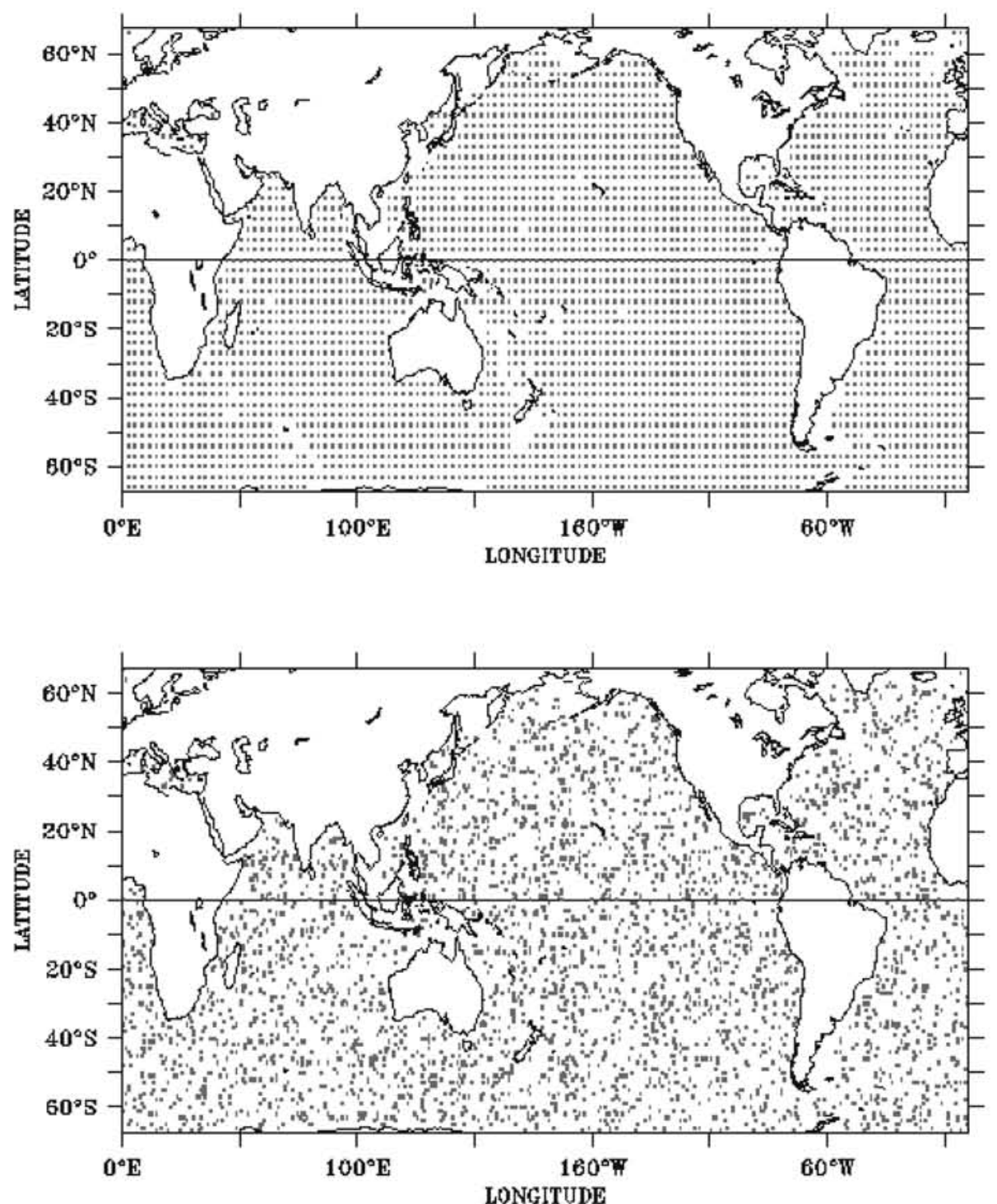

Figure 3. Initial float distribution and float distribution after some time assumed during the ARGO planning phase.

In summary, numerous issues such as the estimation of Ekman transports and the reduction in observational errors in hydrographic data through the implementation of a synoptic observing network will need to be addressed, but if successful, one may expect that space gravity observations will greatly improve our knowledge of the ocean circulation. 


\section{Acknowledgements}

I wish to thank two anonymous reviewers for their help in improving the original manuscript. I gratefully acknowledge Prof. Klees and the Technical University of Delft for having hosted me for a one-year stay during which this paper was written.

\section{References}

Coachman, L.K., and K. Aagaard: 1988, 'Transports through the Bering Strait: Annual and interannual variability', J. Geophys. Res. 93, 15,535-15,539.

Fofonoff, N.P.: 1985, 'Physical properties of seawater: new salinity scale and equation of state for seawater', J. Geophys. Res. 90, 3,332-3,342.

IPCC Third Assessment Report: 2001, 'Climate Change 2001: The Scientific Basis, Contribution of Working Group I to the Third Assessment Report of the Intergovernmental Panel on Climate Change (IPCC)', J. T. Houghton, Y. Ding, D.J. Griggs, M. Noguer, P. J. van der Linden and D. Xiaosu (Eds.), Cambridge University Press, UK. pp 944.

Ganachaud, A., and C. Wunsch: 2000, 'Improved estimates of global ocean circulation, heat transport and mixing from hydrographic data', Nature 408, 453-457.

Ganachaud, A., C. Wunsch, M.-C. Kim, and B. Tapley: 1997, 'Combination of TOPEX/Poseidon data with a hydrographic inversion for determination of the oceanic general circulation and its relation to geoid accuracy', Geophys. J. Int. 128, 708-722.

Hall, M.M., and H.L. Bryden: 1982, 'Direct estimates and mechanisms of ocean heat transport', Deep-Sea Res. 29, 339-359.

LeGrand, P., H. Mercier, and T. Reynaud: 1998, 'Combining T/P altimetric data with hydrographic data to estimate the mean dynamic topography of the North Atlantic and improve the geoid', Ann. Geophys. 16, 638-650.

LeGrand, P.: 2001, 'Impact of the Gravity Field and Steady-State Ocean Circulation Explorer (GOCE) mission on ocean circulation estimates. Volume fluxes in a climatological inverse model of the Atlantic', J. Geophys. Res. 106, 19,597-19,610.

LeGrand, P., E.J.O. Schrama, and J. Tournadre: 2002, 'An inverse modeling estimate of the dynamic topography of the ocean', Geophys. Res. Letters, in press.

Lemoine, F.G., S.C. Kenyon, J.K. Factor, R.G. Trimmer, N.K. Pavlis, D.S. Chinn, C.M. Cox, S.M. Klosko, S.B. Luthcke, M.H. Torrence, Y.M. Wang, , R.G. Wiliamson, E.C. Pavlis, R.H. Rapp, and T.R. Olson: 1998, 'The development of the joint NASA GSFC and the National Imagery and Mapping Agency (NIMA) geopotential model EGM96', : NASA Technical Publication, NASA Technical Publication NASA, Center for Aerospace Information. Hanover, MD.

Milliff, R.F., M.H. Freilich, W.T. Liu, R. Atlas, and W.G. Large: 2001, 'Global ocean surface vector wind observations from space', Observing the Oceans in the 21st Century, p102-119, C.J Koblinsky and N.R. Smith (Eds.), GODAE Project Office and Bureau of Meteorology, Melbourne.

Pond, S., and G.L. Pickard: 1983, 'Introductory dynamical oceanography', 329 pp., Pergamon Press, Oxford.

Schröter, J., M. Losch, and B. Sloyan: 2002, 'Impact of the Gravity Field and Steady-State Ocean Circulation Explorer (GOCE) mission on ocean circulation estimates 2. Volume and heat fluxes across hydrographic sections of unequally spaced stations', J. Geophys. Res. 107, 4-1-4-20.

Sigman, D.M., and E.A. Boyle: 2000, 'Glacial/interglacial variations in atmospheric carbon dioxide', Nature 407, 171-174.

Wunsch, C.: 1978, 'The North Atlantic general circulation west of $50 \mathrm{~W}$ determined by inverse methods', Revs. Geophys. and Space Phys. 16, 583-620. 
Wunsch, C.: 1984, 'An eclectic Atlantic Ocean circulation model. Part I: The meridional flux of heat', J. Phys. Oc. 15, 1521-1531.

Wunsch, C.: 1996, ‘ The Ocean Circulation Inverse Problem', 422 pp., Cambridge Univ. Press, New York.

Address for Offprints: P. LeGrand, Laboratoire de Physique des Oceans, IFREMER, BP 70, F-29280 Plouzane, France, plegrand@ifremer.fr 